\title{
Globe
}

Revue internationale d'études québécoises

\section{Le pouvoir de la parole dans les relations franco-amérindiennes en Nouvelle-France au XVII ${ }^{\mathrm{e}}$ siècle The Power of Speech between the French and the Amerindians in $17^{\text {th }}$ Century New France}

\section{Ana Isabel Valero Peña}

Volume 6, numéro 1, 2003

URI : https://id.erudit.org/iderudit/1000697ar

DOI : https://doi.org/10.7202/1000697ar

Aller au sommaire du numéro

Éditeur(s)

Globe, Revue internationale d'études québécoises

ISSN

1481-5869 (imprimé)

1923-8231 (numérique)

Découvrir la revue

Citer cet article

Valero Peña, A. I. (2003). Le pouvoir de la parole dans les relations franco-amérindiennes en Nouvelle-France au XVII ${ }^{\mathrm{e}}$ siècle. Globe, 6(1), 151-169. https://doi.org/10.7202/1000697ar
Résumé de l'article

Depuis les premiers voyages de découverte, les récits de voyage offrent au lecteur des informations sur les langues des autochtones. Nous avons analysé l'apparition de l'éloquence amérindienne dans les récits de voyageurs et de missionnaires français en Nouvelle-France. Le Français du XVII ${ }^{\mathrm{e}}$ siècle dédaigne la société amérindienne, sans écriture, tout en l’admirant pour son éloquence. Cette rhétorique inattendue et le fait de parler « sans être interrompu » fascinent les voyageurs. Ceux-ci insistent sur l'incompréhension de ces discours; en revanche, les missionnaires essaient d'accommoder leur propre discours évangélisateur à la vie amérindienne. En effet, ils profitent du rôle que joue la parole chez les indigènes pour rendre l'Amérindien apte à la conversion. 


\title{
Le pouvoir de la parole dans les relations franco-amérindiennes en Nouvelle-France au $x{ }^{e}$ siècle
}

\author{
Ana Isabel Valero Peña \\ Université de Barcelone (Espagne)
}

\begin{abstract}
Résumé - Depuis les premiers voyages de découverte, les récits de voyage offrent au lecteur des informations sur les langues des autochtones. Nous avons analysé l'apparition de l'éloquence amérindienne dans les récits de voyageurs et de missionnaires français en Nouvelle-France. Le Français du xvII siècle dédaigne la société amérindienne, sans écriture, tout en l'admirant pour son éloquence. Cette rhétorique inattendue et le fait de parler " sans être interrompu n fascinent les voyageurs. Ceux-ci insistent sur l'incompréhension de ces discours ; en revanche, les missionnaires essaient d'accommoder leur propre discours évangélisateur à la vie amérindienne. En effet, ils profitent du rôle que joue la parole chez les indigènes pour rendre l'Amérindien apte à la conversion.
\end{abstract}

The Power of Speech between the French and the Amerindians in $17^{\text {th }}$ Century New France

Abstract - Since the first voyage of discovery, travel stories offer information about Amerindian languages. We analyze the appearing of Amerindian eloquence in the writings of French travelers and missionaries in New France. The Frenchmen of the $17^{\text {th }}$ century look down to the Amerindian community lacking a written language, but they admire it at the same time for its eloquence. The unexpected rbetoric as well as the right to speak without being interrupted fascinate the travelers, who insist bowever on the incomprebensibility of the Amerindian speech. The missionaries, on the contrary, try to adapt their evangelical sermon to Amerindian life and they take advantage of the importance of speaking amongst Amerindians in order to convert the local population.

[...] quelques vns semblent estre nés à l'eloquence. [...] dans leur grande prudence \& moderation de paroles [...] vous remarquez tousiours vne singuliere douceur \& discretion. Ie n'ay gueres assisté en leurs

Ana Isabel Valero Peña, * Le pouvoir de la parole dans les relations francoamérindiennes en Nouvelle-France au xv1 ${ }^{e}$ siècle ", Globe. Revue internationale d'études québécoises, vol. 6, $\mathrm{n}^{\circ} 1,2003$. 


\section{REVUE INTERNATIONALE D'ÉTUDES QUÉBÉCOISES}

Conseils, mais toutes les fois qu'ils m'y ont inuité i'en fuis sorty auec estonnement sur ce point.

Jean DE BRÉbEuf, $1636^{1}$

Les Hurons, c'était l'aristocratie indienne, hommes sensibles à l'honneur, beaux parleurs et aux attitudes nobles; les Algonquins, quelque chose comme des bourgeois [...]; les Montagnais, la plèbe, frustes au point de savoir à peine parler.

Samuel DE CHAMPLAIN ${ }^{2}$

$\mathrm{Au} \mathrm{XVII}{ }^{\mathrm{e}}$ siècle, les missionnaires français sont chargés d'évangéliser les Amérindiens de l'Amérique du Nord. Ce projet demande une connaissance approfondie de l'Autre. C'est ainsi que, dès les premières années de colonisation en Nouvelle-France, les religieux tentent de s'initier aux cultures huronne, algonquine et montagnaise afin de pouvoir ensuite les convertir. Explorateurs et missionnaires se sont vite aperçus que la société amérindienne utilise les talents des individus les plus éloquents, ce qui nous plonge au cœur d'un des aspects les plus modernes de la réalité amérindienne, la parole continuant à être, presque quatre siècles plus tard, la façon la plus commune de faire entendre sa voix. En effet, lors des voyages des Français en Nouvelle-France, les processus de colonisation et d'évangélisation vont de pair. La prépondérance de la parole chez l'Amérindien est essentielle pour les explorateurs-colonisateurs, et surtout pour les missionnaires. Marcel Trudel écrit à ce propos :

C'est vers 1540 que François $I^{\text {er }}$ met une étiquette religieuse sur ses entreprises américaines ${ }^{3}$. Dans les textes officiels, on ne ratera plus une occasion d'énon-

1. R. G. Thwaites, The Jesuits Relations and Allied Documents, Travels and Explorations of Jesuit Missionnaries in New France, 1660-1671, Cleveland, Burrows Bros, 1896-1901, vol. X, p. 258-260 (toute référence à cette édition des Relations des Jésuites sera dorénavant indiquée par les lettres JR dans le texte).

2. Cité par Jean Riverain [éd.], Dictionnaire des explorations, Paris, Larousse, 1966, p. 63.

3. À propos du mélange exploration-colonisation et évangélisation de la part des Français en Nouvelle-France, voir Joseph Bouchaud, "Évangélisation et 


\section{LE POUVOIR DE LA PAROLE EN NOUVELLE-FRANCE}

cer [...] que la France travaille d'abord à l'augmentation du nom de Dieu. [...] Depuis que le pape en 1493 avait partagé les terres nouvelles entre l'Espagne et le Portugal, il n'y avait pour la France qu'un moyen d'intervenir sans heurter le Saint-Siège : se donner un rôle missionnaire, grâce auquel le pape permettra l'expédition française ${ }^{4}$.

Le discours indigène apparaît dans les premiers récits de voyage comme une des formes de la langue autre. Nous pourrons constater à quel point l'éloquence est admirée par les voyageurs, surtout par les missionnaires, dans leur effort continu pour maîtriser la parole indienne. Pour ce faire, nous analyserons un vaste corpus d'écrits de la NouvelleFrance. Nous étudierons ainsi les textes suivants (dont la date de rédaction originelle est indiquée entre parenthèses) : ceux des explorateurs Jacques Cartier ${ }^{5}$ (1535), Jean de Léry ${ }^{6}$ (1580), Samuel de Champlain ${ }^{7}$ (1604-1629) et Marc Lescarbot ${ }^{8}$ (1611) ; les écrits de l'ursuline Marie de l'Incarnation $^{9}$ (1677), des récollets Gabriel Sagard ${ }^{10}(1632,1636)$, Joseph

colonisation ', Revue française d'bistoire d'outre-mer, vol. LIV, 1967, p. 39-43 et p. 194-197; Donald Smith, Le sauvage pendant la période "béroïque "de la Nouvelle-France. 1534-1663, d'après les historiens canadiens-français des XIX et $X X^{e}$ siècles, Québec, Hurtubise $\mathrm{HMH}, 1974$; Jacques Mathieu, La nouvelle France. Les Français en Amérique du Nord, xve-xvme siècles, Paris, Belin, 1991 et Paolo Carile, "L'eredità di Colombo ", La scoperta dell'America e le lettere francesi, Milano, Cisalpino-Istituto Editoriale Universitario, 1992, p. 11-47.

4. Marcel Trudel, Histoire de la Nouvelle-France, Montréal, Fides, vol. I : Les vaines tentatives (1524-1603), 1963, p. 130-132.

5. Les relations de Jacques Cartier, M. Bideaux léd.], Québec, Les Presses de l'Université de Montréal, 1986.

6. J. Léry, Histoire d'un voyage en terre du Brésil, Lestringant [éd.], Montpellier et Paris, Les Presses du Languedoc/Max Chaleil Éditeur, 1992, chap. XX, p. 223-238.

7. Deux éditions consultées: The Works of Samuel de Champlain, Henri-Perceval Biggar [éd.], 1922-1935; et La France d'Amérique: voyages de Samuel de Champlain, 1604-1629, J. Glénisson [éd.], Paris, Imprimerie Nationale, 1994.

8. M. Lescarbot. Histoire de la Nouvelle-France, Paris, Tross, chap. VII, livre sixième, p. 661-671.

9. Correspondance de Marie de l'Incarnation, Guy Oury [éd.], Paris, Solesme, 1971.

10. Trois éditions consultées pour le Grand voyage au Pays des Hurons: la première est l'originale (Paris, Denys Moreau, 1632), la deuxième date de 1865 (Paris, Tross), la troisième est l'édition critique de $\mathrm{J}$. Warwick (Montréal, les Presses de l'Université de Montréal, 1998). Cette dernière sera désignée GV dans 
Le Caron (1636), Chrestien Le Clercq ${ }^{11}$ (1691) et Louis Hennepin ${ }^{12}$ (1697) ainsi que les relations des jésuites Pierre Biard ${ }^{13}$ (1616), Paul Le Jeune (1633), Jean de Brébeuf ${ }^{14}$ (1636), François du Peron ${ }^{15}$ (1639), Barthélémy Vimont $(1642,1644)$, Paul Ragueneau $(1646,1650)$, François Bressani ${ }^{16}$ (1653), Sébastien Rasles (1723) ${ }^{17}$, Joseph-François Lafitau ${ }^{18}$ (1724) et François-Xavier de Charlevoix ${ }^{19}$ (1744). Pour plus de simplicité, et parce que la parole a joué un rôle similaire dans les deux entreprises, les textes des explorateurs et des missionnaires seront traités indistinctement dans le présent travail.

\section{Les harangues dans la vie amérindienne}

Décrivons pour commencer les circonstances dans lesquelles les harangues sont prononcées par les Amérindiens. Lors de la première rencontre entre Amérindiens et Français, il est courant d'assister à une harangue de bienvenue (" une fois que le vieillard, maitre de la maison, eut fait sa harangue à notre bienvenue ${ }^{20}$ "). Voici un des nombreux extraits où Cartier reproduit la scène du discours prononcé par un Amérindien :

Et lors commança ledit agouhanna à faire ung sermon et preschement comme cy davant est dict estre leur

le texte. Nous utiliserons aussi, de Gabriel Sagard, l'Histoire du Canada (Paris, Denys Moreau, 1636).

11. Chrestien Leclercq, Nouvelle Relation de la Gaspésie, qui contient les Mours $\mathcal{E}$ la Religion des Sauvages Gaspesiens Porte-Croix, adorateurs du Soleil, $\mathcal{E}$ d'autres Peuples de l'Amerique Septentrionale, dite le Canada, Paris, Amable Auroy, (1691a) ; et Chrestien Leclercq, Premier Etablissement de la foy dans la NF, Paris, Amable Auroy, 1691b.

12. Louis Hennepin, Nouvelle découverte d'un très grand pays, Utrecht, Guillaume Broedelet, 1697.

13. P. Biard, JR, op. cit., II, vol. XIII, p. 201 ; vol. III, p. 69, 91.

14. J. Brébeuf, JR, op. cit., vol. X, p. 117-123.

15. F. du Peron, JR, op. cit., vol. XV, p. 155-157.

16. F. Bressani, JR, op. cit., vol. XXXIX, p. 103-105, 119, 121.

17. S. Rasles, JR, op. cit., vol. LXVII, p. 133, 145-147.

18. J.-F. Lafitau, Mours des sauvages américains comparées aux mours des premiers temps, Paris, Saugrain aîne et Hochereau, vol. II, p. 474 et ss.

19. Charlevoix, Histoire et description générale de la Nouvelle-France, Paris, Rollin fils, 1744 .

20. Léry, éd. 1992, p. 175. 
coustume de faire joye et congnoissance en faisant icelluy seigneur chere audit cappitaine et sa compaignye. (Cartier, éd. 1986, p. 151)

Les cérémonies de bienvenue s'accompagnent d'une offrande de présents : lors des échanges de cadeaux, les Amérindiens prononcent aussi des discours de remerciement aux Français. Faute d'un échange compréhensible de paroles, on échange des présents ; c'est une façon de maintenir l'amitié avec les nouveaux venus :

Ce Paraousti fit vne longue harangue tendant à ce que les nôtres allassent en I I sa cabane, et en signe d'amitié bailla sa robbe ou manteau de chamois au conducteur de la trouppe Françoise. (Lescarbot, éd. 1865, vol. I, p. 61-62)

Le récollet Chrestien Leclercq suggère, note $R$. Wilkin ${ }^{21}$, que les " présents " qui ponctuent l'éloquence diplomatique amérindienne (et dont les Français se sont servis pour négocier avec eux ${ }^{22}$ ) remplacent les mots qu'ils sont censés symboliser : "on ne parle enfin, $\&$ on ne répond que par des presens, \& c'est pour cela que dans les harangues, les presens passent pour des paroles ${ }^{23}$ " (Leclercq, 1691b, p. 118). Dans l'extrait qui suit, le discours sert à remercier les Français de leurs cadeaux :

21. Rebecca Wilkin, "Les mots et les choses "aux Hurons" : l'archéologie d'une rencontre •, Religion and French Literature, Buford Norman léd.], Amsterdam/ Atlanta, GA, XI, 1998, p. 61-62.

22. Rebecca Wilkin, op. cit., envoie le lecteur à JR, vol. LI, p. 205 et vol. LXVI, p. 177-179.

23. Voici comment M. Trudel explique ces présents substituts de paroles : “ Ce sont les discours qui ont été prononcés au cours des cérémonies, discours que l'on a appelés paroles. Nous les tenons d'interprètes européens qui les ont traduites à mesure qu'on les a prononcées et qui les ont ensuite exprimées par l'écriture ; au moment de la ratification, un missionnaire (comme c'est le cas dans un traité de 1666 avec les Iroquois) a soin de leur faire lecture en langue iroquoise de ce qui a été écrit. Cette conservation par l'écriture donnait aux Français comme aux Anglais un avantage sur les Amérindiens [...]. Les exemples de ces paroles, au langage fort imagé, abondent dans la documentation historique, grâce aux textes verbatim ou aux résumés que nous ont transmis les Relations des Jésuites. - Marcel Trudel, . Les Hurons et Murray en 1760 * dans Denis Vaugeois, [éd.], Les Hurons de Lorette, Sillery, Septentrion, 1996, p. 133-159, p. 153. 


\section{REVUE INTERNATIONALE D'ÉTUDES QUÉBÉCOISES}

A cette harangue plus eloquent en Algonquin, que ie ne l'ay couchée en François : Paul Atondo repartit encor plus elegamm?t en son lãgage. Mille actiôs de graces, mes freres, de vos presens. (Vimont, 1642, JR, vol. XXII, p. 148)

Les voyages rapportent des discours prononcés dans des situations de tous les jours, par exemple, avant un festin (L. Hennepin, 1697, p. 209). Les textes du récollet $G$. Sagard, considérés comme l'une des meilleures descriptions de la société huronne de l'époque, recèlent de nombreux exemples où des discours sont prononcés. Dans l'extrait qui suit, il s'agit de harangues lors de la pêche ${ }^{24}$ :

En chacune des cabanes de la pesche, il y a vn Predicateur de poisson, qui a accoustumé de les prescher, s'ils sont habiles gens ils sont fort recherchez, por ce qu'ils croyent que les exhortations d'vn habile homme, ont vn grand pouuoir d'attirer les poissons dans leurs rets, comme eux l'eloquence d'vn grand Ciceron à sa volonté. Celuy que nous auions s'estimoit vn des plus rauissans, aussi le foisoit il beau voir demener $\&$ des mains $\&$ de la langue quand il preschoit, comme il faisoit tous les soirs, aprés auoir imposé le silence \& faict ranger vn chacun en sa place, couché de son long, le ventre en haut comme luy. (Sagard, 1636, vol. II, p. 641)

G. Sagard, qui connaissait bien la langue huronne, avoue que, lors des discours, les Amérindiens emploient des mots incompréhensibles pour un Européen. Le passage suivant fait encore référence à la pêche : "Pour auoir bonne pesche ils bruslent aussi du petun, en prononçans de certains mots que ie n'entends pas * (Sagard, 1636, vol. II, p. 64225). La

24. Pour d'autres exemples de déclaration et de discours, lors de la vie quotidienne des Hurons, voir la table de matières du Grand Voyage au Pays de Hurons (éd. 1865).

25. Voir aussi : Sagard, 1636, vol. II, p. 496. Dans les deux cas, le récollet fait référence à un rituel de la culture amérindienne où les * mots incompréhensibles * appartiendraient à un langage codé. Mais en général, les Occidentaux font allusion à la difficulté qu'ils ont subie face à la langue autre ; à ce propos, voir J. Brébeuf, 1636, JR, X, p. 256 et E. Martinell Gifre et al. [éd.], Kassel, Reichenberger, 2000 , p. 140-151. 
guerre, qui fait aussi partie de la vie quotidienne de l'Amérindien ${ }^{26}$, est encore ponctuée de harangues. Sagard rend compte d'une harangue funèbre qui fait appel à la vengeance :

[...] et s'il est mort en guerre, le Capitaine faict vne Harangue en maniere d'Oraison funebre, en la presence du corps, incitant et exhortant l'assemblée, sur la mort du deffunct, de prendre vengeance d'vne telle meschanceté, et de faire la guerre à ses ennemis. (Sagard, 1632, éd. 1865, vol. I, p. 201)

Comme le démontrent ces premiers textes sur la Nouvelle-France, la parole occupe une place dominante dans la vie amérindienne ${ }^{27}$.

\section{"Il n'y a lieu au monde où la Rhétorique soit plus puissante qu'en Canadas "}

Les premiers voyageurs en Nouvelle-France, note Marie-Christine Gomez-Géraud, semblent avoir été sensibles à une forme spectaculaire de l'éloquence indienne ${ }^{28}$, sans doute d'autant plus remarquable que le contenu des discours proférés restait obscur pour les explorateurs. Il suffit de relever, dans les relations de J. Cartier par exemple, les occurrences des termes de " harangue ", "prédication ", "prêche " et "sermon ", qui tous renvoient à des situations de parole publique ${ }^{29}$.

26. Voir Sagard, 1636, vol. II, p. 453. Sur la guerre entre Iroquois et Hurons, voir la Correspondance de l'ursuline Marie de l'Incarnation, notamment la lettre LXI, datant de 1654, éd. 1971.

27. Pour d'autres allusions à la parole de l'Autre dans les écrits sur la NouvelleFrance, voir André Vachon, Éloquence indienne, Montréal, Fides, 1968 ; ainsi que * Harangues, requêtes et adresses de grands chefs ", dans Marguerite Vincent Tehariolina, La nation buronne, son bistoire, sa culture, son esprit, Québec, Pélican et Septentrion, 1984.

28. Pour cette utilisation de la parole remarquée par les Européens, Guy Laflèche (Le missionnaire, le sorcier et l'apostat, Montréal, PUM, 1973) nous renvoie à JR, vol. V, p. 34, 62 et 194 .

29. Marie-Christine Gomez-Géraud, * L'entrée de l'Indien dans la littérature française. Genèse d'un stéréotype ", Revue Europe. L'Invention de l'Amérique, avril 1992 , p. 66. 
En effet, le voyage de J. Cartier inclut maintes allusions à la langue autre sous forme de discours prononcés par les chefs amérindiens : " [...] notre cappitaine et ses gens et lors commança une grande harangue " (Cartier, éd. 1986, p. 142). Ce discours tenu au " cappitaine et à ses gens " dans une langue qu'ils n'entendaient pas, note $M$. Bideaux, instaure une communication aussi problématique que celle que provoquera la lecture de l'Évangile selon saint Jean que fera bientôt Cartier au peuple d'Hochelaga. Le goût de l'Indien pour la parole d'apparat a très tôt frappé les Européens et le père Biard écrit en 1616: "Ces gens, je crois, sont les plus grands harangueurs de toute la terre. Ils ne feront rien sans cela" . Dans le monde amérindien, l'éloquence constitue une des vertus cardinales des chefs et le discours est un élément essentiel de la vie politique et sociale.

L'utilisation de la parole chez l'Amérindien comme critère de gouvernement est considérée par les Français de la colonie comme un phénomène négatif lié à l'absence d'écriture. Dans les premières relations des jésuites, signale $M$. Bideaux ${ }^{30}$, n'apparaît pas l'essentiel de l'organisation politico-sociale des Amérindiens. Les jésuites découvraient une structure sociale trop lâche : les chefs, pour exercer leur autorité, n'avaient que le pouvoir de leur éloquence ${ }^{31}$. Pour illustrer cette idée, laissons place aux mots du jésuite Le Jeune: "Toute l'autorité de leur chef est au bout de ses lèvres, il est aussi puissant qu'il est éloquent " (JR, vol. VI, p. 42). Le même Le Jeune écrivit aussi ce passage, considéré à juste titre comme l'un des plus remarquables discours du jésuite concernant la parole amérindienne ${ }^{32}$ :

30. Michel Bideaux, "Culture et découverte dans les relations des Jésuites ", $x$ Vif siècle, $\mathrm{n}^{\circ} 112,1976$, p. 6.

31. " Car leur capitaine n'est esleu que pour sa langue et il est autant bien obéy qu'il l'a bien pendue. Ils n'ont point d'autres loix que sa parole. " (JR, vol. V, p. 194-195, cité par Margaret Leahey, * "Comment peut un muet prescher l'Évangile ?" Jesuit Missionaries and the native languages of New France ", French Historical Studies, vol. 19, n' 1, 1995, p. 126).

32. Avant de citer les études concernant l'ouvre de Le Jeune, rappelons que d'autres missionnaires avaient auparavant fait allusion au pouvoir de la parole chez l'Amérindien : * [..] qui harangue le mieux est le mieux obey * (Sagard, 1636, vol. II, p. 419). Ainsi, Anne de Vaucher Gravili, analysant l'œuvre du récollet G. Sagard, écrit dans des termes semblables à propos de l'importance de l'éloquence amérindienne: "L'exercice du pouvoir et le fonctionnement des 
Il n'y a lieu au monde où la Rhétorique soit plus puissante qu'en Canadas : $\&$ neantmoins elle n'a point d'autre habit que celuy que la nature luy a baillé : elle est toute nuë \& toute simple, \& cependant elle gouverne tous ces peuples, car leur Capitaine n'est eleu que pour sa langue: \& il est autant bien obey, qu'il l'a bien penduë, ils n'ont point d'autres loix que sa parole. Il me semble que Cicero dit qu'autrefois toutes les nations ont esté vagabondes, \& que l'eloquence les a rassemblées: qu'elle a basti des villes \& des citez. Si la voix des hommes a tant de pouvoir, la voix de l'esprit de Dieu sera-t-elle impuissante ${ }^{33}$ ? (1633, JR, vol. V, p. 194)

L'historien Jean-Marie Therrien parle en ces termes du pouvoir de la parole chez l'Amérindien :

institutions est fondé sur le don de la parole : la harangue, qu'elle soit prière ou exhortation, est l'élément de toute cohésion sociale chez les Amérindiens. . (Anne de Vaucher Gravili, "Le dictionnaire de la langue huronne de Gabriel Sagard, un manuel de civilisation ", La Guirlande di Cecilia, studi in onore di Cecilia Rizza, Porindisi, Schena-Nizet, 1996, p. 496.)

33. * Les récits de voyage en Nouvelle-France mettent en scène d'éloquents capitaines amérindiens et rapportent, en traduction, leurs très nombreuses harangues. Parmi celles-ci, certaines sont courtes et en style indirect, mais d'autres sont si longues qu'elles constituent l'essentiel du récit. C'est dans les Relations du jésuite Le Jeune que les harangues vont prendre une place très importante, à tel point que dès 1637 les Relations se rapprochent d'une anthologie de harangues amérindiennes entrecoupées de commentaires apostoliques ", Carmen Mata Barreiro, "La traduction du matériel sémiotique des communications interculturelles dans les relations de voyages en Nouvelle-France •, Études canadiennes/ Canadian Studies, $\mathrm{n}^{\circ} 41,1996$, p. 21 . À propos de la rhétorique (et/ou éloquence) chez Le Jeune, voir Normand Doiron, "Rhétorique jésuite de l'éloquence sauvage au $\mathrm{Xvi}^{\mathrm{e}}$ siècle. Les "relations" de Paul Le Jeune (1632-1642)", $X V f^{\circ}$ siècle, $\mathrm{n}^{\circ} 173$, oct.-déc. 1991, p. 375-402; Rémi Ferland, Les relations des Jésuites. Un art de la persuasion (Procédés de rbétorique et fonction conative dans les "Relations - du Père Le Jeune), Québec, Les Éditions de la Huit, 1992 ; Pierre Berthiaume, "Fleurs de rhétorique montagnaise: fleurs vénéneuses ", dans Amérindia, $\mathrm{n}^{\circ} 17,1992$, p. 121-148 ; ainsi que les divers articles de Réal Ouellet, dont : : Entreprise missionnaire et ethnographie dans les premières Relations de Paul Le Jeune ", dans G. Demerson léd.], Les Jésuites parmi les bommes. $x v^{e}-x v f^{e}$ siècles, Clermond-Ferrand, Bibliothèque municipale et interuniversitaire, 1985, p. 93-104 et, en collaboration avec Marie Parent, " Mise en scène et fonctions de la parole amérindienne dans les relations de voyage *, Transferts culturels et métissages Amérique/Europe, L'Harmattan, 1996, p. 281-304. 
La société amérindienne, comme toute société d'ailleurs, ne peut se passer de porte-parole [...]. L'originalité de la société amérindienne est d'utiliser les talents des individus les plus éloquents pour le bien-être de tous. "Parlez, chefs, mais à la place des autres et pour les autres!" La parole des chefs, comme les cadeaux qui l'expriment, est un outil par lequel la société amérindienne construit son identité et cherche à se conserver quand elle est menacée $\mathrm{e}^{34}$.

\section{Modération de paroles dans des cœurs aigris}

Les récits de voyage de la Nouvelle-France sont des témoignages précieux sur une altérité jusque-là inconnue. Un des critères des écrivains-voyageurs pour définir les indigènes réside dans la faculté de s'exprimer. Ainsi, ceux qui ne sont pas de bons orateurs ne seraient pas " sensibles à l'honneur " et ceux qui occupent le lieu le plus bas dans la pyramide sociale sont "frustres au point de savoir à peine parler ". Les Européens sont étonnés de l'usage de la parole chez les Amérindiens, car la rhétorique est normalement réservée aux "civilisés ${ }^{35}$ ". De même, ils s'étonnent de l'absence d'écriture dans la culture des Autochtones. Ainsi, la parole est d'un côté admirée pour son pouvoir éloquent et, de l'autre, discréditée par l'absence de support écrit. C'est surtout cette absence d'écriture qui placerait les langues nouvelles du côté de la barbarie $^{36}$. Ne pas écrire n'empêchait évidemment pas les Indiens de transmettre leurs légendes de génération en génération, par la tradition orale, comme le souligne Elie de Comminges ${ }^{37}$. Toutefois, les écrits du

34. Jean-Marie Therrien, Parole et pouvoir: figure du chef amërindien en Nouvelle-France, Montréal, l'Hexagone, 1986, p. 110.

35. "Bref, dira Brébeuf, c'estoit chose digne d'estonnement de voir dans des cœeurs aigris vne telle moderation de paroles " (1636, JR, vol. X, p. 262).

36. Il est connu que les langues amérindiennes sont considérées barbares, mais d'un autre côté, les missionnaires, premiers dialectologues en Nouvelle-France, ont souvent remarqué la richesse de ces langues; à ce propos, voir $\mathbf{J}$. Brébeuf, 1636, JR, vol. X, p. 54, G. Sagard, 1636, vol. II, p. 338 et 364, Le Clercq, 1691a, p. 162 et J.F. Lafitau, 1724, vol. II, p. 488.

37. Elie de Comminges, "Les récits de voyage des jésuites en NF : la mission du Père Biard (1611-1613) ", French Review, vol. 49, n 6, 1976, p. 840-855. 
jésuite $P$. Ragueneau montrent que cette façon de conserver les traditions n'est à ses yeux pas crédible si on la compare avec la parole vraie du Français dont témoignent les livres saints. La parole amérindienne est ici discréditée par le paganisme, c'est une parole sans foi qui va devenir fable :

Le plus ancien Capitaine des assistans [...] dit que luy va raconter la pure verité, $\&$ comment il est arriué que la terre, qui estoit sumergée dans les eaux, en ait esté poussée dehors $[\ldots]$. Ce bon Chrestien $[. .$.$] ayant donné$ quelque temps audience à la fable de ce Capitaine infidèle [...] dit : mais voyant que tu ne racontes que des fables, qui n'ont point de fondement que le mensonge, i'ay plus de droit de parler que toy. Où sont les escritures qui nous fassent foy de ce que tu dis? Estant permis à vn chacun de controuuer ce qu'il voudra, estce merueille que nous ne sçachions rien de veritable, puisque nous deuons auouër que les Hurons ont esté menteurs de tout temps? Mais les François ne parlent point par coeur, ils conjeruent de toute antiquité les liures Saints, où la parole de Dieu mesme est escrite, sans qu'il soit permis à aucun d'y alterer le moins du monde [...]. (1646, JR, vol. XXX, p. 60-62)

Il y a malgré cela des cas où le fait de ne pas connaître l'écriture rend l'éloquence indienne encore plus digne d'admiration :

[...] non hanno ne libri, ne scritture, i negotij si trattano per ambasciate, [...] le conclusioni prese ne' consegli, e mille altre particolarità, che noi non sapressimo ridire senza scriuere. [...] Intendono, e discorrono bene, hanno [...] grand' eloquenza ${ }^{38}$ [...]. (F. Bressani, 1653, JR, vol. XXXVIII, p. 260)

38. "Ils n'ont pas de livres, ni d'écriture, on traite les affaires dans des réunions, [...] [ils reprennent] les conclusions des Conseils ainsi que mille autres particularités que nous ne saurions pas dire à nouveau sans les avoir écrites auparavant. [...] Ils comprennent et parlent bien et ils ont de la grandiloquence [...] , notre traduction. 


\section{REVUE INTERNATIONALE D'ÉTUDES QUÉBÉCOISES}

Dédaigner une société sans écriture et admirer en même temps son éloquence (" mille autres particularités que nous ne saurions pas dire à nouveau sans les avoir écrites auparavant "), voilà le paradoxe occidental vis-à-vis de la parole amérindienne. Les Amérindiens parlent peu, note Lucien Campeau ${ }^{39}$, jamais deux à la fois, ils n'interrompent jamais leur interlocuteur et ne se laissent pas interrompre; ils ne dialoguent pas ${ }^{40}$, ils font des discours et il n'est jamais question de leur conversation, mais de leur éloquence.

Abordant le sujet de l'éloquence, Lescarbot décrit la façon de parler des Amérindiens en se basant sur deux caractéristiques du discours, soit parler posément et parler sans être interrompu :

[...] ils parlent fort II posément, comme se voulans bien faire entendre, et s'arrêtent aussi-tôt en songeant vne grande espace de temps, puis reprennent leur parole. Ils vsent bien souvent de cette façon de faire parmi leurs harangues au conseil, où il n'y a que les plus principaux, qui sont les anciens. Les femmes et enfans n'assistent point ${ }^{41}$. (Lescarbot, éd. 1865, vol. I, p. 282)

39. Lucien Campeau, La première mission d'Acadie, Québec, PUL et Rome, MHSJ, 1967, p. 246.

40. Campeau renvoie le lecteur à JR, vol. V, p. 26 et VI, p. 206. Voir aussi Sagard, 1636, vol. II, p. 424 et léry, éd. 1992, p. 136. Cet effet ne se produit pas dans d'autres textes où les auteurs présentent de véritables dialogues, à thème religieux, entre l'Amérindien et l'Occidental. Nous ne mentionnerons que les exemples de deux jésuites, les dialogues entre Le Jeune et Pierre Pastedechouan ou ses commensaux de l'hiver 1634-1635 et le * Diálogo sobre a conversão do gentio * de Manuel de Nóbrega (1557?), dans Monumenta Brasiliae, S. Leite, Rome, 1956. Voir R. Ferland, 1992, op. cit., p. 186-196 et Eni Pulcinelli Orlandi, "Les discours fondateurs de la brésilienneté ", Naissance du Brésil moderne, 1998 , p. $131-142$.

41. La plupart des écrits sur la Nouvelle-France insistent en effet sur ces caractéristiques du discours amérindien : ne pas interrompre son interlocuteur, parler posément. Pour ce qui est de la première, voir $\mathrm{S}$. de Champlain, éd. 19221935, vol. V, p. 63 ; L. Hennepin, 1697, p. 209 ; Lafitau, 1724, vol. I, p. 483 ; ibid., II, p. 88. Les Occidentaux demeurent donc "ébranlez" ("il prononça son discours si serieusement, que nous gens furent ébranlez *, écrit L. Hennepin) face au discours de l'Amérindien : le "barbare "bouscule donc le "civilisé " dans des récits où il n'y a plus lieu d'utilisier la dénomination " barbare "pour désigner 
Le jésuite Brébeuf souligne pour sa part le fait que les Amérindiens prononcent leurs discours lentement et en utilisant beaucoup de métaphores ${ }^{42}$, deux aspects qui contribuent à l'incompréhension des langues amérindiennes :

[...] la maniere de parler [dans les Conseils], laquelle à cause de sa diuersité a vn nom -different, \& s'appelle acwentonch; elle est commune à tous les Sauuages; ils haussent \& flechissent la voix comme d'vn ton de Predicateur à l'antique, mais lentement, posément, distinctement [...]. I'ay autrefois ouy dire à quelque Truchement, que ces Nations icy auoient vn langage particulier en leurs Conseils; mais i'ay experimenté le contraire; ie sçay bien qu'ils ont quelques termes particuliers, ainsi qu'on a en toutes sortes d'arts [... $\}$. Il est vray que leurs discours sont d'abord difficiles à entendre, à cause d'vne infinité de Metaphores [...]. (1636, JR, vol. X, p. 256)

La présence de métaphores dans les discours amérindiens est un aspect remarqué à plusieurs reprises dans les récits de voyage ${ }^{43}$. Chez certains Occidentaux, l'usage de métaphores contribue à la vision négative des langues indigènes : elles seraient langues de la passion, riches

l'Autochtone. À propos de la façon posée de parler des Amérindiens, voir Lescarbot, éd. 1865, vol. I, p. 277. Pour ce qui est de la place donnée au silence dans les harangues amérindiennes, voir Mata Barreiro, op. cit., 1996, p. 20.

42. Les observateurs noteraient le caractère codé, réglé, complexe de la prise de la parole. Cela ne confirme pas le désordre de la barbarie, car règles et codes sont indices de civilisation. Il s'agit de la parole dans les Conseils où l'absence de monarchie n'est pas synonyme d'anarchie: ce sont des peuples sans roi, certes, mais non pas sans lois. Le langage métaphorique est un langage recherché, propre à un peuple " complexe ", sophistiqué.

43. Voir Brébeuf, 1636, JR, vol. X, p. 218. Les Amérindiens, notait R. Toupin, ont une manière très imagée de parler, par exemple : " mettre la chaudière sur le feu, lever la hache *, c'est déclarer la guerre. - Renverser la chaudière ', c'est suspendre les hostilités. (Arpents de neige et Robes noires. Brève relation sur le passage des Jésuites en NF aux xVIf et xVIT siècles., Saint-Laurent, Québec, Bellarmin, 1990.) Pour ce qui est de l'emploi des métaphores dans le discours de l'indigène dans l'œuvre de Hennepin, voir Catherine Broué, "Modalités et fonctions de la parole amérindienne dans la Nouvelle Découverte de Louis Hennepin ", Revista Española de Estudios Canadienses, vol. 3, $\mathrm{n}^{\circ} 2$, p. 26-39, notamment p. 34. 


\section{REVUE INTERNATIONALE D'ÉTUDES QUÉBÉCOISES}

en métaphores, et non comme les langues raisonnables (européennes), riches en abstractions ${ }^{44}$. Mais la parole de l'Amérindien est aussi utilisée par l'Occidental. Il fait dire à l'Indien ce qu'il prétend déclarer dans son récit. C'est ainsi qu'accédant au statut d'objet littéraire, déclare M.-C. Gomez-Géraud ${ }^{45}$, l'orateur indigène acquiert autorité. Dépouillé de ses caractéristiques ethniques, il devient un outil à des fins d'efficacité rhétorique. En effet, l'indigène, démentant tout préjugé européen, apparaît dès les premières pages du premier tome de l'Histoire de la NouvelleFrance de Lescarbot comme un être s'exprimant "avec beaucoup de jugement ". Sous le regard de l'Europe triomphe seul le Sauvage littéraire :

Et neantmoins noz peuples de la Nouvelle-France ne sont si brutaux, stupides ou lourdaux que l'on pourroit penser ${ }^{46}$. Et trouve que c'est à grand tort qu'on dit d'eux que ce sont des betes, gens cruels et sans raison. Car je n'y ay point veu de niais ${ }^{47}$ comme il s'en trouve quelquefois és paîs de l'Europe : ils parlent avec beaucoup de jugement [...]. (Lescarbot, éd. 1865, vol. I, p. 7)

L'auteur de l'Histoire de la Nouvelle-France ne prétend pas par là valider son récit, mais plutôt rejeter un préjugé.

Les religieux, fort amateurs de rhétorique, assistent aux conseils des Amérindiens et se les font traduire par ceux-ci. Une fois de plus, l'éloquence indienne l'emporte sur celle des missionnaires et leur ignorance des langues indigènes :

44. A. Pagden, The Fall of Natural Man, Cambridge, Cambridge University Press, 1982 , p. 185, cité par Aurore Monod-Becquelin, "La parole et la tradition orale amérindiennes dans les récits des chroniqueurs aux $\mathrm{XVI}^{\mathrm{e}}$ et $\mathrm{XVII}^{\mathrm{e}}$ siècles , Amérindia, n $^{\circ} 6,1984$, p. 335.

45. M.-C. Gomez-Géraud, op. cit., p. 68.

46. Quarante ans plus tard, le jésuite $P$. Ragueneau soutient que les indigènes sont des barbares et il fait pourtant l'éloge de leur éloquence : * Voila la harangue que fit ce Capitaine Huron, ie n'y adjouste rien, \& mesme ie n'y puis ioindre la grace que luy donnoit le ton de sa voix, \& les regards de son visage. La nature a son eloquence, E quoy qu'ils soient Barbares, jls n'ont pas dépoüillé ny l'estre d'homme, ny la raison, ny vne ame de mesme extraction que les nostres *. (1650, JR, vol. XXXVI, p. 220) (Nous soulignons.)

47. Pour d'autres adjectifs qualifiant les indigènes, voir Maurice de la Porte, Les Epithetes [françoises], Paris, Gabriel el Buon, 1571 ; Slatkine Reprints, 1973, p. 45. 
Voyla le Sommaire de la harangue de ce Sauuage [Estienne Pigroüich ${ }^{48}$ ], dit le Pere Brebeuf, qui nous a donné ces memoires, ie suis extremément marry, adiouste-il que ie ne puisse repeter mot à mot tout ce qu'il dit, mais ny ie n'ay peu le bien comprendre, ny ne l'ay peu bien sçauoir des interpretes, lesquels apres auoir repeté ce que dessus, dirent qu'il n'estoit pas possible de redire ce qu'il auoit dit, \& qu'eux \& tous ceux qui se mesle[nt] de parler la langue des Sauuages ne font que begayer en comparaison de cét homme, \& qu'il auoit aussi bien dit, comme le Pere de Bressany venoit de bien prescher. Ce que ie puis dire, c'est que sa façon, sa deuotion, \& toute son action toucha extremément tous les François \& tous les Sauuages. (B. Vimont, 1644, JR, vol. XXV, p. 256)

Aux yeux des missionnaires, imbus de culture classique, les Indiens paraissent avoir hérité l'éloquence des anciens Romains ${ }^{49}$. Tite-Live, Cicéron et Aristote deviennent ainsi des modèles oratoires dans les relations des jésuites Brébeuf (JR, vol. X, p. 244) et Le Jeune :

Ce Capitaine prend la parole pour respondre, mais avec une rhetorique aussi fine et déliée, qu'il en sçauroit sortir de l'escolle d'Aristote, ou de Ciceron. Il gagna au

\footnotetext{
48. Elsie McLeod Jury affirme : a Pigarouich était un shaman algonquin et chrétien apostat dont le père Buteux dit: jamais ouy Sauvage mieux parler ny plus hardiment qu'il fit en Eglise l'espace d'un quart d'beure. Les jésuites reconnurent que Pigarouich pouvait faire beaucoup pour favoriser ou pour entraver le progrès du christianisme parmi les Indiens. Il n'est plus question de lui après 1644 . Elsie McLeod Jury, "Estienne Pigroüich „, dans Dictionnaire Biographique du Canada, Laval, Les Presses de l'Université Laval, 1966, vol. I, p. 562 et ss.

49. Voir M. Bideaux, 1976, op. cit., p. 23 ; R. Ferland, 1992, op. cit., p. 173; Normand Doiron, L'art de voyager. Le déplacement à l'époque classique, SainteFoy, Presses de l'Université Laval/Paris, Klincksieck, 1995; * La rhétorique robine de l'bistoria nuda et le récit de voyage ", La France-Amérique, 1998, op. cit., p. 382-392 ; et Carmen Mata Barreiro, "Traduction et représentations de l'Autre: ré-énonciation et parole des Amérindiens dans les relations de voyage des Français en Nouvelle-France, dans Les chemins du texte, VI Coloquio da APFFUE (Santiago, 19, 20 e 21 de febreiro de 1997), Universidade de Santiago de Compostela, Asociación de Profesores de Filología Francesa de la Universidad Española, 1998, p. 408-418.
} 
commencement de son discours la bienveillance de tous les François par une profonde humilité, qui paroissoit avec bonne grace dans ses gestes et dans ses paroles. (1633, JR, vol. V, p. 204)

En comparant le parler amérindien et celui de l'Antiquité, les missionnaires mettent l'accent sur l'idéal de civilisation que devraient suivre les Autochtones.

\section{"Celuy qui sçauroit leur langue} les manierait comme il voudroit "

S'exprimer " elegamm?t . (B. Vimont) ou "dans une simplicité sincere "(J. Le Caron) sont donc les principales caractéristiques du discours amérindien qui étonnent l'Occidental dès les premiers voyages. L'utilisation de la parole par les indigènes est étroitement liée aux intérêts des Occidentaux dans la colonie. Il en est ainsi pour cet extrait de l'Histoire du Canada où, comme dans d'autres récits, hiverner parmi les Amérindiens est perçu comme une pratique devant servir à améliorer les rapports entre Occidentaux is: Autochtones:

C'est pourquoy le sieur Chãplain au cõmencement du mois de Iuillet 1629. despescha vn François auec quelques Barbares, vers la nation des Abenaquioue peuples habitans du costé du Sud de l'habitation, [...] Ce François estant là arriué, les fit haranguer par son Truchement, de la part du Gouuerneur de Kebec, \& demãder s'ils leur pourroient nourrir quelque François iusques au commencement de l'Esté prochain, \& ce faisant ils les obligeroient à contracter amitié auec eux, \& les maintenir à l'encontre de leurs ennemis. Les Albenaquioue ayans ouy la harangue de ce Truchement, tindrent cõseil, \& conclurent à la faueur des François [...]. (Sagard, 1636, vol. IV, p. 979-980) 
De même, dans l'extrait qui suit, $M$. Lescarbot rapporte le discours de Messamoet afin de louer les effets de la colonisation ${ }^{50}$ :

Messamoet commence à haranguer devant les Sauvages
[...] qu'ils pourroient facilement domter leurs ennemis
s'ils se vouloient entendre et se servir de l'amitié des
François [...] Somme il fut prés d'vne heure à parler
avec beaucoup de vehemence et d'affection et avec vn
contournement de corps et de bras tel qu'il est requis
en vn bon Orateur. (Lescarbot, éd. 1865, vol. II, p. 534)

La façon idéale de parler serait donc d'avoir recours aux méthodes des grands orateurs : sans interruption en utilisant la force de la parole accompagnée des gesticulations.

En somme, l'attitude des missionnaires au sujet de l'apprentissage des langues indiennes est la suivante: ils visent en premier lieu à s'approprier les langues, pour pouvoir ensuite connaître leur rhétorique et s'en emparer afin de convaincre ultimement les Autochtones de se convertir. Le besoin d'apprendre des langues indigènes pour évangéliser provient de l'importance donnée au message oral par les missionnaires, mais aussi du pouvoir de la parole dans les sociétés amérindiennes. M. Leahey emprunte une des phrases les plus célèbres du jésuite Le Jeune à ce propos : "Comment peut un muet prescher l'Évangile? „ et il écrit :

Le Jeune, after only a few weeks in Canada, bad become convinced that someone who knew the Indians languages well would be all-powerful among them (" tout puissant parmy eux "). [...] This being the case, Le Jeune would try yet another strategy to master the language : a winter with the Montagnais ${ }^{51}$.

50. N'oublions pas que M. Lescarbot apparaît comme l'un des grands défenseurs de la colonisation en Nouvelle-France. À propos de l'exaltation du projet colonisateur selon les traductions des discours amérindiens, voir $\mathrm{S}$. de Champlain, éd. 1994, p. 72-73, 276-277 et 284.

51. Margaret Joan Leahey, 1995, op. cit., p. 126. 
M. Leahey ira jusqu'à souscrire à la thèse de Le Jeune, selon laquelle " il n'y a lieu au monde où la thétorique soit plus puissante qu'en Canadas ". Les missionnaires vont en tenir compte et se servir du pouvoir de l'éloquence pour convertir les indigènes. P. Carile affirme à ce propos :

L'opera di conversione si fondava sulla parola. La padronanza delle lingue amerindie unita a quella delle tecniche retoriche in cui erano maestri, permetteva ai Gesuiti di presentare i fondamenti teologici del Cattolicesimo e di argomentare, dal loro punto di vista, gli errori delle concezioni e delle pratiche religiose autoctone ${ }^{52}$.

Effectivement, les missionnaires reconnaissaient que seule l'éloquence pouvait remettre le monde à l'endroit, comme le rappelle R. Wilkin ${ }^{53}$. L'œuvre de R. Ferland ${ }^{54}$ prétend montrer que les relations constituent une vaste entreprise de séduction et préciser ce dont elles cherchent à persuader et comment elles cherchent à le faire ${ }^{55}$. Les procédés de rhétorique n'auraient d'intérêt que dans cette optique :

Dans un premier temps [...], il faudrait captiver les "coeurs \& courages" amérindiens par leurs propres langues. Après s'être initiés au troc des mots par la maîtrise des vocabulaires Montagnais, Algonquins, et Hurons (entre autres), les missionnaires espéraient

52. P. Carile, 1992, op. cit. : "La conversion est fondée sur la parole. La maîtrise des langues amérindiennes unie à celle des techniques rhétoriques qu'ils dominaient, permettait aux Jésuites de présenter les fondements théologiques du catholicisme et d'argumenter, d'après leur point de vue, les erreurs des conceptions et des pratiques religieuses autochtones *. Notre traduction.

53. R. Wilkin, 1998, op. cit., p. 71.

54. R. Ferland, 1992, op. cit., p. 11.

55. Une autre stratégie de conversion de la part des missionnaires est l'utilisation de l'image. À ce propos, voir François-Marc Gagnon, La conversion pour l'image, un aspect de la mission des Jésuites auprès des Indiens du Canada au XVIf siècle, Montréal, Bellarmin, 1975; François Weil, "Conversions et baptêmes en NF (xvil ${ }^{\mathrm{e}} \mathrm{xv} \mathrm{II}^{\mathrm{e}}$ siècles), principe, méthodes, résultats ", dans $\mathrm{C}$. Blanckaert, [éd.], Naissance de l'ethnologie, Paris, Cerf, 1985. 


\section{LE POUVOIR DE LA PAROLE EN NOUVELLE-FRANCE}

dominer l'éloquence amérindienne afin de subjuguer la raison - Sauvage ${ }^{56}$.

Ce n'est donc pas par hasard si c'est dans la relation du jésuite Le Jeune que se trouve un des dialogues les plus marquants des relations; la force de la parole devient incalculable dans les relations missionnaire-néophyte: " [...] i'ay bien d'autres veritez plus grandes à vous dire quand ie sçauray parler " (Le Jeune, 1634, JR, vol. VII, p. 94). Les missionnaires n'oubliaient, à aucun moment, que "celui qui harangue le mieux et le mieux obéi " et pensaient qu'ils pouvaient, par là, "fléchir et plier les volontés " des Amérindiens.

56. R. Wilkin, 1998, op. cit., p. 57. 